\title{
Training needs for Ugandan primary care health workers in management of respiratory diseases: a cross sectional survey
}

\author{
CURRENT STATUS: ACCEPTED \\ BMC Health Services Research $\triangle$ BMC Series \\ Rebecca Nantanda \\ Makerere University Lung Institute \\ \rnantanda@gmail.comCorresponding Author
}

Gerald Kayingo

University of California, Davis

Rupert Jones

University of Plymouth

Frederik van Gemert

Groningen University

Bruce Kirenga

Makerere University College of Health Sciences

DOI:

10.21203/rs.2.15713/v1

\section{SUBJECT AREAS}

Health Economics \& Outcomes Research Health Policy

\section{KEYWORDS}

respiratory diseases, primary care, health workers, knowledge, skills 
Abstract

Background Acute and chronic respiratory diseases are among the leading causes of morbidity and mortality in Uganda, but there is little attention to chronic respiratory diseases in the health programmes. This survey assessed the gaps in knowledge and skills, among healthcare workers in managing respiratory illnesses.

Methods A mixed methods study was conducted among primary care health workers, physicians and healthcare planners to assess gaps in knowledge and skills, and training needs in managing respiratory illnesses. The perspectives of patients with respiratory diseases were also sought. Data were collected using questionnaires, patient panel discussions and review of pre-service training curricula for clinicians, nurses and midwives. Survey Monkey was used to collect survey data. Descriptive analysis was undertaken for quantitative data and content analysis for qualitative data. Results A total of 104 respondents participated in the survey and of these, $76.9 \%(80 / 104)$ were primary care health workers, $16.3 \%$ (17/104) specialist clinicians and 6.7\% (7/104) healthcare planners. Over $90 \%$ of the respondents indicated that more than half of the patients in their clinics presented with respiratory symptoms. More than half $(52 \%)$ of the primary care health workers were not comfortable in diagnosing and managing chronic respiratory diseases like asthma and COPD. Only $4 \%$ of them were comfortable performing procedures like pulse oximetry, nebulization, and interpreting $\mathrm{x}$-rays. Majority (75\%) of the primary care health workers had received in-service training but only $4 \%$ of the sessions focused on respiratory diseases. The clinicians indicated that the preservice training curricula included a wide scope of respiratory diseases, but the actual training had not sufficiently prepared them to manage respiratory diseases. The patients were unsatisfied with the care in primary care facilities and reported that they were often treated for the wrong illnesses. Conclusions Respiratory illnesses contribute significantly to the burden of diseases in primary care facilities in Uganda. Management of patients with respiratory diseases remains a challenge partially because of inadequate knowledge and skills of the primary care health workers. A training programme to improve the competences of health workers in respiratory medicine is highly recommended. 
Full Text

Due to technical limitations, full-text HTML conversion of this manuscript could not be completed. However, the manuscript can be downloaded and accessed as a PDF.

Figures

\section{Heal th workers' skills in management of chronic respiratory diseases}

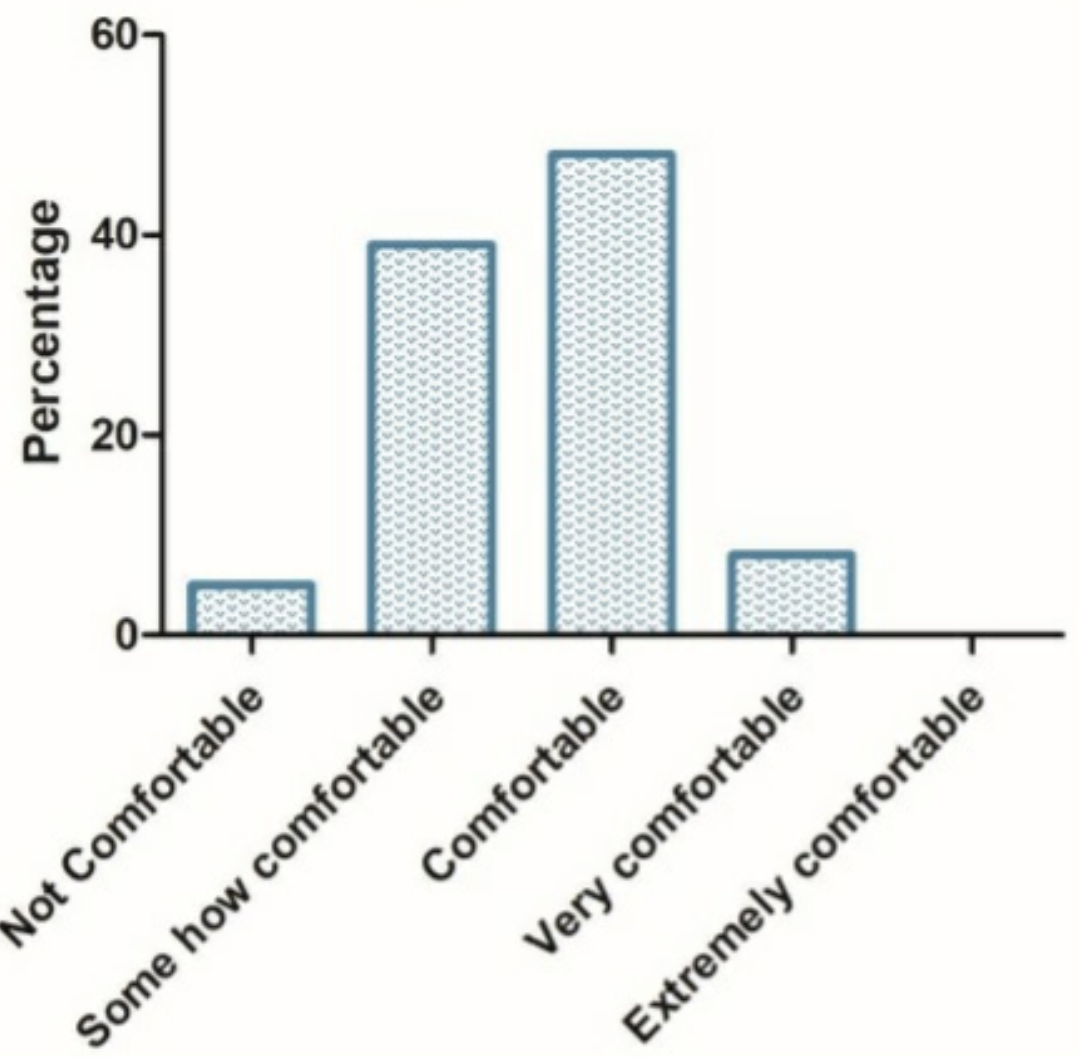

Figure 1

Knowledge and skills of health workers in managing respiratory diseases 


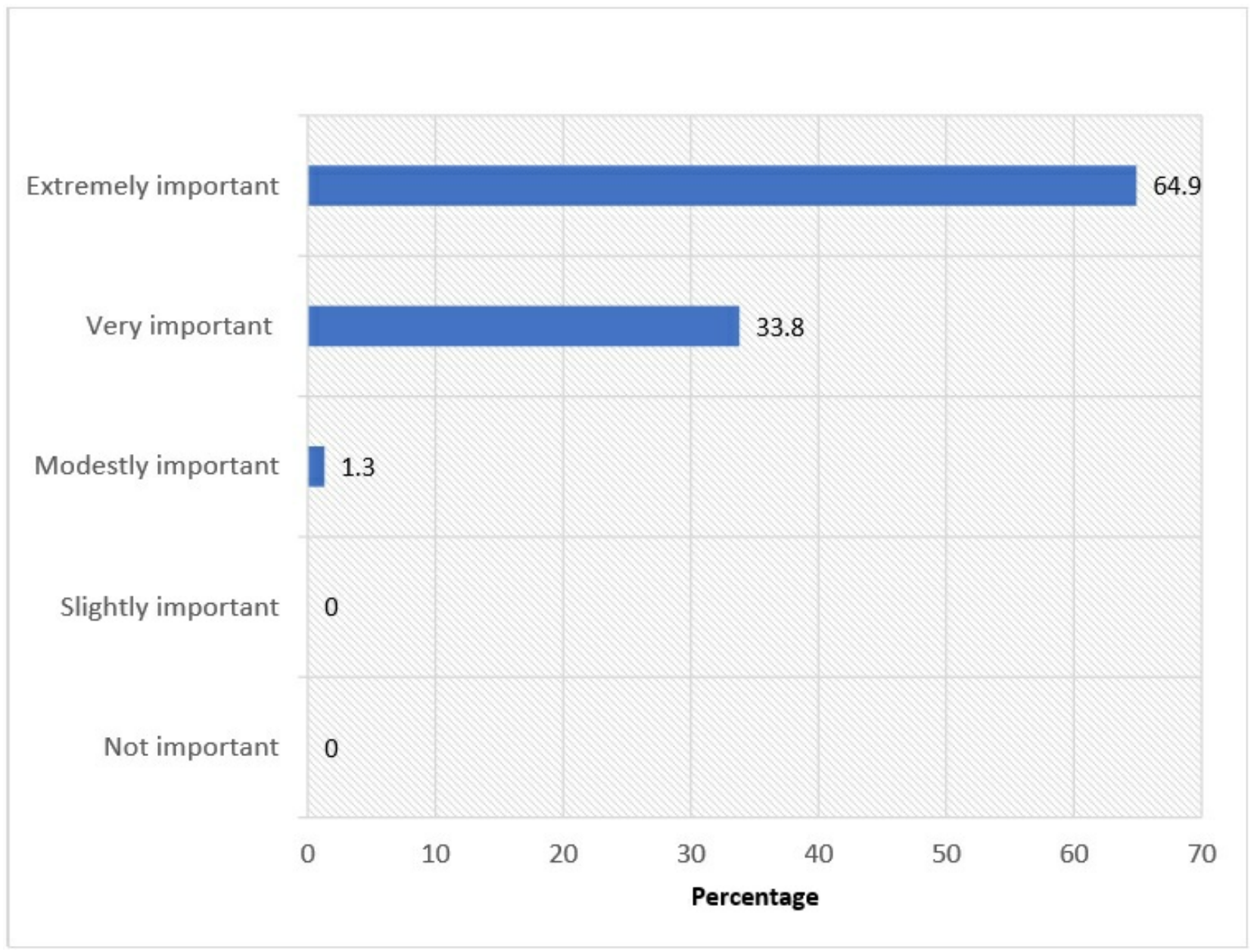

Figure 2

Importance of the respiratory medicine in-service training programme 